\title{
Diagnostic Value of Metagenomic Next-Generation Sequencing of Bronchoalveolar Lavage Fluid for the Diagnosis of Suspected Pneumonia in Immunocompromised Patients
}

\author{
Pengcheng Lin \\ Department of Pulmonary and Critical Care Medicine, The First Affiliated Hospital of Wenzhou Medical University, Wenzhou, Zhejiang \\ Yi Chen \\ Department of Infectious Diseases, The First Affiliated Hospital of Wenzhou Medical University, Wenzhou, Zhejiang \\ Shanshan Su \\ Department of Pulmonary and Critical Care Medicine, The First Affiliated Hospital of Wenzhou Medical University, Wenzhou, Zhejiang \\ Wengang Nan \\ Department of Pulmonary and Critical Care Medicine, The First Affiliated Hospital of Wenzhou Medical University, Wenzhou, Zhejiang \\ Lingping Zhou \\ Department of Pulmonary and Critical Care Medicine, The First Affiliated Hospital of Wenzhou Medical University, Wenzhou, Zhejiang \\ Ying Zhou \\ Department of Pulmonary and Critical Care Medicine, The First Affiliated Hospital of Wenzhou Medical University, Wenzhou, Zhejiang \\ Yuping Li ( $\square$ wzliyp@163.com ) \\ Department of Pulmonary and Critical Care Medicine, The First Affiliated Hospital of Wenzhou Medical University, Wenzhou, Zhejiang
}

\section{Research Article}

Keywords: Metagenomic next-generation sequencing, Conventional microbiological tests, Bronchoalveolar lavage fluid, Diagnostic performance, Immunocompromised,

Posted Date: January 3rd, 2022

DOI: https://doi.org/10.21203/rs.3.rs-1201244/v1

License: (c) This work is licensed under a Creative Commons Attribution 4.0 International License. Read Full License 


\section{Abstract}

Background: To evaluate the diagnostic value of metagenomic next-generation sequencing (mNGS) of bronchoalveolar lavage fluid (BALF) in immunocompromised patients for the diagnosis of suspected pneumonia in comparation to that of conventional microbiological tests (CMTs).

Methods: Sixty-nine immunocompromised patients with suspected pneumonia received both CMTs and mNGS of BALF were analyzed retrospectively. The diagnostic value was compared between CMTs and mNGS, using the clinical composite diagnosis as the reference standard.

Results: Sixty patients were diagnosed of pneumonia including fifty-two patients with identified pathogens and eight patients with probable pathogens. The overall detection rate of mNGS for pathogens were higher than that of CMTs. However, a comparable diagnostic accuracy of mNGS and CMTs were found for bacterial and viral infections. mNGS exhibited a higher diagnostic accuracy for fungal detection than CMTs (78\% vs. $54 \%, P]$ 0.05), which mainly because of the high sensitivity of mNGS in patients with Pneumocystis jirovecii pneumonia (PJP) (100\% vs. 28\%, P凶0.05). Among fifty-two patients with definite pathogens, nineteen patients (37\%) were identified as pulmonary mixed infection, mNGS test showed a higher detection rate and broader spectrum for pathogen detection than that of CMTs in mixed infection. Moreover, Pneumocystis jirovecii was the most common pathogen in mix infection and mNGS have identified much more co-pathogens of PJP than CMTs.

Conclusions: mNGS of BALF improved the microbial detection rate of pathogens and exhibited remarkable advantages in detecting PJP and identifying mixed

infections in immunocompromised patients.

\section{Background}

Pneumonia is the most common cause of morbidity and mortality in immunocompromised patients, for which early identification of causative pathogens is necessary (1). However, the conventional microbiological tests (CMTs) methods are time consuming and represent a low rate of positive detection, which can not meet the diagnosis needs of an immunocompromised patient. Even with the combination of culture and culture-independent techniques (nucleic acid amplification testing and serological testing), the aetiological diagnosis of severe pneumonia in immunocompromised patient is usually unclear.

Metagenomic next-generation sequencing (mNGS), an unbiased pathogen detection method, has been used in infectious diseases increasingly in recent decades. It is mainly used for assessing the sterile body fluids in the clinical setting, including cerebrospinal fluid, joint effusion and blood(2-4). For non-sterile body fluids, such as bronchoalveolar lavage fluid (BALF), the applications of mNGS are greatly limited, especially for immunocompromised patients. Pan et al explored the application of mNGS of BALF in thirteen immunocompromised patients for the diagnosis of pneumonia and showed improved detection of opportunistic pathogens(5). However, peng et al reported a similar diagnostic value of mNGS of BALF in immunocompromised patients to the CMTs for all types of pathogens(6).

In this study, we attempted to evaluate the diagnostic performance of mNGS of BALF for pneumonia in immunocompromised patients compared with that of CMTs. Moreover, we intended to show diagnostic accuracy rates for different types

of pathogens.

This study received approval from the Ethics Committee of the First Affiliated Hospital of Wenzhou Medical University (No. 2020-111) and was conducted in accordance with the Declaration of Helsinki (as revised in 2013). Informed consent to publish the information were obtained from the personal patients.

\section{Patients And Methods}

\section{Patients}

From September 2018 to December 2020, all immunocompromised patients with suspected pneumonia admitted to the First Affiliated Hospital of Wenzhou Medical University were retrospectively investigated.

The inclusion criteria of patients were as follows: (1) at least eighteen years old; (2) had an immunocompromised condition;(3) admitted to the Department of Pulmonary and Critical Care Medicine due to suspected pneumonia;(4) had mNGS of BALF within $48 \mathrm{~h}$ after admission, and other relevant samples available for the conventional microbiological tests in standard procedures;

Immunocompromised status(7) ( $\geq 1$ of the following risk factors were found): (1) hematologic cancer;(2) chemotherapy during the last three month; (3) chronic steroid ( $>0.3 \mathrm{mg} / \mathrm{kg} / \mathrm{d}$ of prednisone-equivalent for $\geq 3$ weeks) or biologic drug use for autoimmune diseases or other immunosuppressive therapy;(4) solid-organ transplant receipt during the last six months;(5) neutropenia;(6) acquired or inherited severe immunodeficiency;

Patients with suspected pneumonia meet the both criteria below: (1) new-onset fever, cough, expectoration or dyspnea; (2) new-onset abnormal chest imaging manifestations; 


\section{Methods \\ Data collection}

The following data were recorded: age, sex, underlying diseases and clinical manifestations, illness severity (pneumonia severity index (PSI) score), chest radiology, results of laboratory examination (including microbiological testing) and patient outcomes (regular clinic follow-up or telephone conversation).

\section{Microbiological tests}

All patients underwent the operation of bronchoalveolar lavage following a standard safety protocol (8). The BALF specimens were divided into aliquots. One aliquot was set for bacterial and fungal smear (including Gomori methenamine silver (GMS) staining and acid-fast stain) and culture. One aliquot was submitted for the detection of galactomannan antigen and cryptococcus capsular polysaccharide antigen. X-pert MTB/RIF detection of DNA sequences specific for Mycobacterium Tuberculosis (MTB) and real-time polymerase chain reaction (PCR) for cytomegalovirus (CMV) and influenza $A / B$ virus. The other aliquots $\left(5 \mathrm{~mL}\right.$, settled in a sterile sputum container, stored at $\left.-20^{\circ} \mathrm{C}\right)$ were sent to $\mathrm{BGI}-\mathrm{Huada}$ Genomics Institute (Shenzhen, China) for detection.

For the other samples, urine was submitted for the detection of antigen of Legionella pneumophila and Streptococcus pneumoniae. Peripheral blood samples were sent for the detection of the galactomannan antigen to Aspergillus, Cryptococcus capsular polysaccharide antigen to Cryptococcus and immunoglobulin $\mathrm{G}$ and $\mathrm{M}$ antibodies to parainfluenza, adenovirus, CMV, Chlamydia pneumoniae and Mycoplasma pneumoniae using commercial enzyme-linked immunosorbent assays (Ani Labsystems) according to the manufacturer's instructions.

\section{mNGS of BALF}

The procedure of mNGS for BALF samples includes Nucleic acid extraction, Library construction, Sequencing, and Bioinformatic Analyses according to previous studies $(9,10)$.

\section{Criteria for a Positive mNGS Result}

1. Bacteria (mycobacteria excluded), virus and parasites:

A microbe was considered clinically significant microbes (CSMs) when its coverage rate scored 10-fold greater than that of any other microbes according to Miao's study(11).

2. Fungi:

A microbe was considered CSMs when its coverage rate scored 5-fold greater than that of any other fungus due to its low biomass in DNA extraction $(12,13)$.

3. Mycobacteria:

MTB was defined as positive when at least 1 read was mapped to either the species or genus level because of the low possibility for contamination and difficulty of DNA extraction(14). Nontuberculous mycobacteria (NTM) were considered when the mapping read number was in the top 10 in the bacteria list because of the low possibility for contamination (15) and low yield rate (16).

Regardless of coverage rate, oral commensals were not defined as CSMs unless they were deemed to be significant by the physicians or proven otherwise(6).

Coverage rate was regarded as the measurement parameter in our study because of the consideration of confounding factors such as pathogen genome size, nucleic acid contamination and total number of sequencing reads (12).

\section{Clinical composite diagnosis as the reference standard}

The medical records such as clinical features, laboratory examination, microbiological tests (including mNGS and CMTs), chest imaging and therapeutic response, were reviewed independently by the two physicians who specialize in the management of infection in immunocompromised hosts to determine whether the patients had infectious aetiology or not and identify the pathogens (definite or probable). An in-depth discussion was performed when there was any disagreement between the two physicians and another senior physician was consulted if consensus could not be reached.

\section{Statistical analysis}

SPSS 22.0 (IBM Corporation) was used for analysis. Continuous variables were reported as the mean \pm standard deviation (SD) or the median (25th, 75th percentiles) depends on they were normally distributed or non-normal distribution. Categorical variables were presented as numbers (percentages). Determination of microbiological aetiology and clinical composite diagnosis were used as the reference standard. The chi-square test, 
McNemar's test or Fisher's exact test was used to compare the diagnostic performance of mNGS and CMTs. All tests were two-tailed and $P<0.05$ was considered significant.

\section{Results}

\section{Patient characteristics}

A total of 69 patients including 44 male (64\%) were enrolled in this study. The mean age was(57士15) years. Thirty-three patients (48\%) received a longterm corticosteroid therapy for solid-organ transplantation or autoimmune diseases and twenty-eight patients (41\%) treated with chemotherapy for solid tumors or hematologic malignancy. The median PSI score was(127 \pm 51$)$. Twenty- two patients (32\%) received invasive mechanical ventilation and 25 of 69 patients developed septic shock. The clinical features of all patients including demographic characteristic, laboratory findings and prognosis were shown in Table 1.

Table 1

Characteristic of 69 immunocompromised patients

\section{Characteristic}

Age (year), mean $\pm S D$

Gender, male, n (\%)

Immunocompromised status, n (\%)

Hematologic malignancy

Solid-organ transplantation

Solid tumour receiving chemotherapy

Immunosuppressive therapy ${ }^{\mathrm{a}}$

Prolonged corticosteroid therapy ${ }^{b}$

Disease severity

$\mathrm{PSI}$ score, mean \pm SD

Invasive Mechanical ventilation, $\mathrm{n}(\%)$

Septic shock, n (\%)

Laboratory findings

WBC $\left(10^{9} / \mathrm{L}\right)$, median (Q1, Q3)

Neutrophils $\left(10^{9} / \mathrm{L}\right)$, median (Q1, Q3)

Lymphocyte count $\left(10^{6} / \mathrm{L}\right)$, median (Q1, Q3)

$\mathrm{PaO}_{2} / \mathrm{FiO}_{2}$, mean $\pm \mathrm{SD}$

Albumin (g/L), mean $\pm S D$

Serum creatinine(mmol/L), median (Q1, Q3)

LDH (U/L), median (Q1, Q3)

CRP (mg/l), median (Q1, Q3)

PCT (ng/ml), median (Q1, Q3)

outcome

Total 30-day mortality, n (\%)

\section{9 patients}

$57 \pm 15$

44(64)

14(20)

5(7)

21(30)

33(48)

$127 \pm 51$

22(32)

25(36)

$6.58(4.93,11.05)$

$5.66(3.23,9.39)$

$0.73(0.35,1.17)$

$196 \pm 98$

$30 \pm 7$

$80(57,127)$

432(304, 726)

$88(42,149)$

$0.27(0.11,1.20)$

14(20)

Notes: a: more than 2 weeks. Underlying diseases include Dermatomyositis (2), Leukemia (1), connective tissue diseases(6), Solid-organ transplantation (9), Hemophilia (1), Chronic nephritic syndrome (1), Thrombocytopenia (1). b. Defined as $>0.3 \mathrm{mg} / \mathrm{kg} / \mathrm{d}$ of prednisone-equivalent for $\geq 3$ weeks. Underlying diseases include Dermatomyositis (1), Connective tissue diseases(9), Solid-organ transplantation (10), Hemophilia (1), Nephrotic syndrome (6), Thrombocytopenia (1), interstitial lung disease(1), Hematologic malignancy (2), Intracranial tumour(1), Dermatology(1).

Abbreviations: PSI: pneumonia severity index; WBC: White blood cell; LDH: lactate dehydrogenase; CRP: C-reactive protein; PCT: procalcitonin; According to the retrospective review of medical records including mNGS and CMTs, 60 patients were diagnosed with pneumonia including 52 patients with definite pathogens and 8 patients with probable pathogens. Another 9 patients were considered non-infection disease including rejection 
of bone marrow with lung involvement $(n=2)$, connective tissue diseases $(n=3)$ or drug $(n=2)$ related interstitial lung disease, lymphoma with lung involvement $(n=1)$ and lung cancer $(n=1)$.

\section{Comparison of mNGS and CMTs Pathogen Detection Methods}

Among 60 patients of confirmed pneumonia, the total detection rate of pathogens by CMTs and mNGS was 26/60 (43\%) and 49/60 (82\%), respectively. The detection rate reached $54 / 60$ (90\%) with a combination of mNGS and CMTs. With regard to bacteria and fungi infection, the detection rate of mNGS was $20 / 25(80 \%)$ and $31 / 34(91 \%)$ respectively, which were significantly higher than that of CMTs (9/25 (36\%) and 15/34 (44\%)) $(P<$ 0.05). No significant difference was found in the detection rate of this two methods for virus infection( $P \otimes 0.05)$. The comparison of different classes of pathogens detection by the CMTs and mNGS was shown in Figure 1.

\section{Comparison of Diagnostic Performance of mNGS and CMTs}

With regard to bacterial detection, the diagnostic sensitivity and specificity of CMTs were $36 \%$ and $82 \%$ respectively. While, mNGS exhibited a diagnostic sensitivity of $80 \%$ and a specificity of $73 \%$. mNGS had a higher diagnostic accuracy than CMTs but not statistically significantly different (75\% vs. $65 \%, P=0.23$ ). A comparable diagnostic accuracy of mNGS and CMTs was also found for viral infections ( $P=0.63)$. However, mNGS exhibited a higher diagnostic accuracy for fungal detection than CMTs (78\% vs. 54\%, $P \otimes 0.05)$, which mainly because of the high sensitivity of mNGS in patients with Pneumocystis jirovecii (100\% vs. $28 \%, P \otimes 0.05)$. GMS staining was positive for Pneumocystis jirovecii in 7 out of 25 patients with Pneumocystis jirovecii pneumonia (PJP), corresponding to a sensitivity of $28 \%$ (95\% Cl 16\%-28\%). The poor sensitivity for the diagnosis of PJP could be improved to $88 \%$ with a combination of GMS staining and Serum $(1,3)$-b-D-glucan (BDG), however, the sensitivity and specificity were still lower than that of mNGS. Comparison of diagnostic performance of mNGS and CMTs for the different types of pathogens were showed in Table 2.

Table 2

Comparison of diagnostic performance of mNGS and CMTs in immunocompromised patients

\begin{tabular}{|c|c|c|c|c|c|c|c|c|c|c|}
\hline & CMTs & & & & & mNGS & & & & \\
\hline & Sensitivity\% & Specificity\% & PPV\% & NPV\% & Accuracy\% & Sensitivity\% & Specificity\% & PPV\% & NPV\% & Accuracy \% \\
\hline & $(95 \% \mathrm{Cl})$ & $(95 \% \mathrm{Cl})$ & $\begin{array}{l}(95 \% \\
\mathrm{Cl})\end{array}$ & $\begin{array}{l}(95 \% \\
\mathrm{Cl})\end{array}$ & $(95 \% \mathrm{Cl})$ & $(95 \% \mathrm{Cl})$ & $(95 \% \mathrm{Cl})$ & $\begin{array}{l}(95 \% \\
\mathrm{Cl})\end{array}$ & $\begin{array}{l}(95 \% \\
\mathrm{Cl})\end{array}$ & $(95 \% \mathrm{Cl})$ \\
\hline $\begin{array}{l}\text { Bacterial } \\
\text { pneumonia }\end{array}$ & $36(21-50)$ & $82(73-90)$ & $\begin{array}{l}53(31- \\
74)\end{array}$ & $\begin{array}{l}69(62- \\
76)\end{array}$ & $65(54-76)$ & $80(63-92)$ & $73(63-79)$ & $\begin{array}{l}63(49- \\
72)\end{array}$ & $\begin{array}{l}87(75- \\
94)\end{array}$ & $75(63-84)$ \\
\hline $\begin{array}{l}\text { Fungal } \\
\text { pneumonia }\end{array}$ & $44(31-57)$ & $63(50-75)$ & $\begin{array}{l}54(38- \\
69)\end{array}$ & $\begin{array}{l}54(43- \\
64)\end{array}$ & $54(41-66)$ & $91(79-98)$ & $66(54-72)$ & $\begin{array}{l}72(63- \\
77)\end{array}$ & $\begin{array}{l}89(73- \\
97)\end{array}$ & $78(66-85)$ \\
\hline $\begin{array}{l}\text { Pneumocystis } \\
\text { jirovecila }^{\text {ing }}\end{array}$ & $28(16-28)$ & $\begin{array}{l}100(91- \\
100)\end{array}$ & $\begin{array}{l}100(58- \\
100)\end{array}$ & $\begin{array}{l}71(66- \\
71)\end{array}$ & $74(66-74)$ & $\begin{array}{l}100(86- \\
100)\end{array}$ & $86(79-86)$ & $\begin{array}{l}81(70- \\
81)\end{array}$ & $\begin{array}{l}100(90- \\
100)\end{array}$ & $91(82-91)$ \\
\hline $\begin{array}{l}\text { Pneumocystis } \\
\text { jirovecil }^{b}\end{array}$ & $88(72-97)$ & $84(75-89)$ & $\begin{array}{l}76(62- \\
83)\end{array}$ & $\begin{array}{l}93(83- \\
98)\end{array}$ & $86(74-92)$ & $\begin{array}{l}100(86- \\
100)\end{array}$ & $86(79-86)$ & $\begin{array}{l}81(70- \\
81)\end{array}$ & $\begin{array}{l}100(90- \\
100)\end{array}$ & $91(82-91)$ \\
\hline $\begin{array}{l}\text { Aspergillus } \\
\text { spp. }\end{array}$ & $86(63-99)$ & $97(92-98)$ & $\begin{array}{l}75(41- \\
87)\end{array}$ & $\begin{array}{l}98(94- \\
100)\end{array}$ & $96(88-98)$ & $57(23-71)$ & $98(95-100)$ & $\begin{array}{l}80(33- \\
99)\end{array}$ & $\begin{array}{l}95(92- \\
97)\end{array}$ & $94(87-97)$ \\
\hline $\begin{array}{l}\text { Viral } \\
\text { pneumonia }\end{array}$ & $53(34-53)$ & $\begin{array}{l}100(95- \\
100)\end{array}$ & $\begin{array}{l}100(64- \\
100)\end{array}$ & $\begin{array}{l}\text { 89(84- } \\
89)\end{array}$ & $90(81-90)$ & $93(70-100)$ & $83(77-85)$ & $\begin{array}{l}61(46- \\
65)\end{array}$ & $\begin{array}{l}98(90- \\
100)\end{array}$ & $86(75-88)$ \\
\hline
\end{tabular}

Notes: ${ }^{a}$ : detect by Gomori methenamine silver staining only; ${ }^{\text {b. }}$ detect by a combination of Gomori methenamine silver staining and serum (1, 3)-bD-glucan( $\geq 80 \mathrm{ng} / \mathrm{l}$ was defined as positive);

Abbreviations: mNGS: metagenomic next-generation sequencing; CMTs: conventional microbiological tests; PPV: positive predictive value; NPV: negative predictive value

\section{Distribution of Pathogens}

The pathogen distribution of 52 patients with identified pneumonia was shown in Figure 2. For the 52 patients with identified pathogens, 33 (63\%) were infected by a single pathogen, while 19 (37\%) were mixed infection (including 9 cases with 2 pathogens, 9 cases with 3 pathogens and 1 case with more than 3 pathogens). The detection rate of mNGS for mixed infection was significantly higher than that of CMTs (79\% vs. $42 \%, P<0.05)$ and more co-pathogens could be identified by mNGS. Fungi-virus-bacteria and fungi-virus coinfection were the most common co-pathogens observed in mix-infection patients. The most common pathogen in mixed infections was Pneumocystis jirovecii $(9 / 19,47.4 \%)$ and Cytomegalovirus (10/19, 52.6\%) (Figure 3).

The pathogens detected isolated by mNGS test including 1 case of Escherichia coli, 1 case of Staphylococcus, 4 cases of Pseudomonas aeruginosa, 16 cases of Pneumocystis jirovecii, 4 cases of Cytomegalovirus, 1 case of Adenovirus, 1 case of Epstein-Barr virus, 2 cases of Chlamydia psittaci, 2 cases of Legionella and 8 cases of mixed infection. For CMTs, 2 cases of Aspergillus, 3 cases of Cryptococcus, 1 case of Mycobacterium tuberculosis 
complex, 1 case of Influenza $A$ and 1 case of mixed infection were detected, which were not identified by mNGS test. Among the 3 cases of cryptococcosis detected by CMTs (PT32, PT40, PT69), the serum and BALF Cryptococcus capsular antigen tests were positive and Cryptococcus neoformans was identified by BALF culture in 2 cases. Pneumocystis jirovecii was identified by mNGS in PT32 and it was considerd as respiratory tract colonization at last in combination with relevant clinical data including serum BDG test and imaging. PT69 was diagnosised as mixed infection of Cryptococcus and Aspergillus, however, none of which was detected by mNGS test (Additional file 1).

\section{Discussion}

Although many researches have evaluated the performance of mNGS in different patients of different infection type(11, 17), there is still lack of studies on the utility of mNGS of BALF in immunocompromised hosts with pneumonia. In a multicenter prospective study of 329 severe communityacquired pneumonia (SCAP) patients, a higher detection rate (up to 90.3\%) and an earlier detection for pathogens by mNGS of BALF than that of CMTs was reported(7). However, immunocompromised cases represented only less than $20 \%$ of the patients enrolled. Similarly, pan et al reported an improved detection for opportunistic pathogens by mNGS of BALF in 13 immunocompromised patients(5). On the contrary, peng et al showed a similar diagnostic performance of mNGS of BALF to CMTs for all types of pathogens in 60 immunocompromised patients(6). In this study, a higher microbial detection rate was showed in immunocompromised patients for using mNGS test of BALF than that of CMTs. For the diagnostic accuracy rate, there was no significant difference in bacteria and viruses' infections by using these two methods. However, mNGS had a higher diagnostic accuracy than CMTs for fungal infections, which was not consistent with the study of Peng, mainly because of the low-proportion of aspergillus which was reported to be difficult for detection by mNG test and high-proportion of pneumocystis in fungal infections in this study.

Pneumocystis jirovecii was the most common opportunistic pathogen in immunocompromised patients(18). It was reported to be responsible for $61.2 \%$ of confirmed pneumonia in immunocompromised patients (6) and $44.6 \%$ of SCAP in immunocompromised patients in a prospective study(7). Compare to CMTs, mNGS showed remarked advantages for detecting Pneumocystis jirovecii. Wang etc (19) showed significantly higher sensitivity of mNGS test than GMS alone for diagnosis PjP (100\% vs. $30.7 \%)$. In the present study, the poor sensitivity of GMS for diagnosis PJP have be remarkably improved to $88 \%$ with the addition of serum BDG test. However, it was still lower than that of mNGS for diagnosis PJP. These results suggest that mNGS is a useful diagnostic tool with good performance for the diagnosis of PJP.

More polymicrobial infections were found in immunocompromised patients in comparison with immunocompetent patients(20). Peng etc(6) reported that more than half of microbiologically confirmed pneumonia having mixed infections. Pathogen detected by CMTs methods is usually a single one, while mNGS can detect multiple pathogens at the same time because of its unbiased detection technology, which has more advantages in the diagnosis of mixed infections $(19,21)$. In this study, 19 patients $(37 \%)$ were identified as pulmonary polymicrobial infections. mNGS showed a higher detection rate and broader spectrum for pathogen detection than that of CMTs in mixed infection. Moreover, Pneumocystis jirovecii was found to be the most common pathogen in pulmonary mixed infection, which was similar to the previous study(22). Compare to CMTs, mNGS have identified much more co-pathogens of PJP. These results indicated that a remarkable advantage of mNGS method for identify mix pulmonary infection and detect co-pathogens of PJP than CMTs.

Rencently, an amount of cases of Chlamydia psittaci infection have been reported(23,24). In our study, we have diagnosed 2 patients of Chlamydia psittaci infection solely by mNGS because of the lack of CMTs for Chlamydia psittaciand they were improved with a proper treatment. This result shows mNGS is also useful in the case of limited CMTs.

However, it was still a challenge for mNGS to identify Aspergillus or Cryptococcus because of the difficulty of DNA extraction from the thick polysaccharide cell wall $(13,25)$. In our study, the diagnosis sensitivity for IPA by CMTs is a litter higher than that of mNGS. As for cryptococcosis, the sensitivity arrived $100 \%$ by using CMTs, while only 1 case was identified by mNGS.

Our retrospective study contains certain limitations. Firstly, as a retrospective study, there were selection bias and recall bias which was inevitable. In addition, the lack of RNA sequencing and partial of PCR methods had failed to evaluate the diagnostic value of CMTs and mNGS better especially for virus infection. The interpretation of virus detected by mNGS was depended on the clinician's subjective judgment more than diagnostically confirmed. Finally, it was difficult to distinguish pathogens from colonization to infection due to the unbiased detection of mNGS without unified standard.

\section{Conclusion}

mNGS using BALF improved the microbial detection rate of pathogens espically in the case of limited CMTs and exhibited remarkable advantages in detecting PJP and identifying mixed infections in immunocompromised patients. However, it is still a challenge for mNGS using BALF to detect Aspergillus or cryptococcus infection.

\section{Declarations}

\section{Ethics approval and Consent to participate}

This study received approval from the Ethics Committee of the First Affiliated Hospital of Wenzhou Medical University (No. 2020-111) and was conducted in accordance with the Declaration of Helsinki (as revised in 2013). Informed consent to publish the information were obtained from the 
personal patients.

\section{Funding}

This work was supported by the Project of National Natural Science Foundation of China (Grant number 81970066) and the Project of Wenzhou Science \& Technology Bureau (Grant number Y20210144). The funding body had no role in the study design, the collection, analysis or interpretation of the data.

\section{Competing interests}

The authors report no conflicts of interest in this work.

\section{Availability of data and materials}

The data that support the findings of this study are available from the corresponding author on reasonable request.

\section{Authors' contributions}

All authors contributed to data analysis, drafting and revising the article, approved the final manuscript, and agreed to be accountable for all aspects of the work.

\section{Consent for publication}

Not Applicable.

\section{Acknowledgments}

Not Applicable.

\section{References}

1. Azoulay E, Mokart D, Kouatchet A, Demoule A, Lemiale V. Acute respiratory failure in immunocompromised adults. Lancet Respir Med. 2019;7(2):173-86.doi:10.1016/S2213-2600(18)30345-X

2. Guo LY, Feng WY, Guo X, Liu B, Liu G, Dong J. The advantages of next-generation sequencing technology in the detection of different sources of abscess. J Infect. 2019;78(1):75-86.doi:10.1016/j.jinf.2018.08.002

3. Chen B, Chen Z, Yang YS, Cai GL, Xu XJ, Guan HZ, et al. Next-generation sequencing combined with serological tests based pathogen analysis for a neurocysticercosis patient with a 20-year history:a case report. BMC Neurol. 2021;21(1):236.doi:10.1186/s12883-021-02277-7

4. Li H, Gao H, Meng H, Wang Q, Li S, Chen H, et al. Detection of Pulmonary Infectious Pathogens From Lung Biopsy Tissues by Metagenomic NextGeneration Sequencing. Front Cell Infect Microbiol. 2018;8:205.doi:10.3389/fcimb.2018.00205

5. Pan T, Tan R, Qu H, Weng X, Liu Z, Li M, et al. Next-generation sequencing of the BALF in the diagnosis of community-acquired pneumonia in immunocompromised patients. J Infect. 2019;79(1):61-74.doi:10.1016/j.jinf.2018.11.005

6. Peng JM, Du B, Qin HY, Wang Q, Shi Y. Metagenomic next-generation sequencing for the diagnosis of suspected pneumonia in immunocompromised patients. J Infect. 2021;82(4):22-7.doi:10.1016/j.jinf.2021.01.029

7. Wu X, Li Y, Zhang M, Li M, Zhang R, Lu X, et al. Etiology of Severe Community-Acquired Pneumonia in Adults Based on Metagenomic NextGeneration Sequencing: A Prospective Multicenter Study. Infect Dis Ther. 2020;9(4):1003-15.doi:10.1007/s40121-020-00353-y

8. Meyer KC, Raghu G, Baughman RP, Brown KK, Costabel U, du Bois RM, et al. An official American Thoracic Society clinical practice guideline: the clinical utility of bronchoalveolar lavage cellular analysis in interstitial lung disease. Am J Respir Crit Care Med. 2012;185(9):100414.doi:10.1164/rccm.201202-0320ST

9. Zhang P, Chen Y, Li S, Li C, Zhang S, Zheng W, et al. Metagenomic next-generation sequencing for the clinical diagnosis and prognosis of acute respiratory distress syndrome caused by severe pneumonia: a retrospective study. PeerJ. 2020;8:e9623.doi:10.7717/peerj.9623

10. Li Y, Sun B, Tang X, Liu YL, He HY, Li XY, et al. Application of metagenomic next-generation sequencing for bronchoalveolar lavage diagnostics in critically ill patients. Eur J Clin Microbiol Infect Dis. 2020;39(2):369-74.doi:10.1007/s10096-019-03734-5

11. Miao Q, Ma Y, Wang Q, Pan J, Zhang Y, Jin W, et al. Microbiological Diagnostic Performance of Metagenomic Next-generation Sequencing When Applied to Clinical Practice. Clin Infect Dis. 2018;67(suppl_2):S231-S40.doi:10.1093/cid/ciy693

12. Schlaberg R, Chiu CY, Miller S, Procop GW, Weinstock G, Professional Practice C, et al. Validation of Metagenomic Next-Generation Sequencing Tests for Universal Pathogen Detection. Arch Pathol Lab Med. 2017;141(6):776-86.doi:10.5858/arpa.2016-0539-RA

13. Bittinger K, Charlson ES, Loy E, Shirley DJ, Haas AR, Laughlin A, et al. Improved characterization of medically relevant fungi in the human respiratory tract using next-generation sequencing. Genome Biol. 2014;15(10):487.doi:10.1186/s13059-014-0487-y 
14. Simner PJ, Miller S, Carroll KC. Understanding the Promises and Hurdles of Metagenomic Next-Generation Sequencing as a Diagnostic Tool for Infectious Diseases. Clin Infect Dis. 2018;66(5):778-88.doi:10.1093/cid/cix881

15. van Ingen J, Kohl TA, Kranzer K, Hasse B, Keller PM, Katarzyna Szafranska A, et al. Global outbreak of severe Mycobacterium chimaera disease after cardiac surgery: a molecular epidemiological study. Lancet Infect Dis. 2017;17(10):1033-41.doi:10.1016/S1473-3099(17)30324-9

16. Ozcolpan OO, Surucuoglu S, Ozkutuk N, Cavusoglu C. [Distribution of nontuberculous mycobacteria isolated from clinical specimens and identified with DNA sequence analysis]. Mikrobiyol Bul. 2015;49(4):484-93.doi:10.5578/mb.9698

17. Gu W, Miller S, Chiu CY. Clinical Metagenomic Next-Generation Sequencing for Pathogen Detection. Annu Rev Pathol. 2019;14:31938.doi:10.1146/annurev-pathmechdis-012418-012751

18. Chen J, He T, Li X, Wang X, Peng L, Ma L. Metagenomic Next-Generation Sequencing in Diagnosis of a Case of Pneumocystis jirovecii Pneumonia in a Kidney Transplant Recipient and Literature Review. Infect Drug Resist. 2020;13:2829-36.doi:10.2147/IDR.S257587

19. Wang J, Han Y, Feng J. Metagenomic next-generation sequencing for mixed pulmonary infection diagnosis. BMC Pulm Med. 2019;19(1):252.doi:10.1186/s12890-019-1022-4

20. Sun T, Wu X, Cai Y, Zhai T, Huang L, Zhang Y, et al. Metagenomic Next-Generation Sequencing for Pathogenic Diagnosis and Antibiotic Management of Severe Community-Acquired Pneumonia in Immunocompromised Adults. Front Cell Infect Microbiol. 2021;11:661589.doi:10.3389/fcimb.2021.661589

21. Chen Y, Feng W, Ye K, Guo L, Xia H, Guan Y, et al. Application of Metagenomic Next-Generation Sequencing in the Diagnosis of Pulmonary Infectious Pathogens From Bronchoalveolar Lavage Samples. Front Cell Infect Microbiol. 2021;11:541092.doi:10.3389/fcimb.2021.541092

22. Jiang J, Bai L, Yang W, Peng W, An J, Wu Y, et al. Metagenomic Next-Generation Sequencing for the Diagnosis of Pneumocystis jirovecii Pneumonia in Non-HIV-Infected Patients: A Retrospective Study. Infect Dis Ther. 2021;10(3):1733-45.doi:10.1007/s40121-021-00482-y

23. Chen X, Cao K, Wei Y, Qian Y, Liang J, Dong D, et al. Metagenomic next-generation sequencing in the diagnosis of severe pneumonias caused by Chlamydia psittaci. Infection. 2020;48(4):535-42.doi:10.1007/s15010-020-01429-0

24. Su S, Su X, Zhou L, Lin P, Chen J, Chen C, et al. Severe Chlamydia psittaci pneumonia: clinical characteristics and risk factors. Ann Palliat Med. 2021;10(7):8051-60.doi:10.21037/apm-21-1502

25. Clarke EL, Lauder AP, Hofstaedter CE, Hwang Y, Fitzgerald AS, Imai I, et al. Microbial Lineages in Sarcoidosis. A Metagenomic Analysis Tailored for Low-Microbial Content Samples. Am J Respir Crit Care Med. 2018;197(2):225-34.doi:10.1164/rccm.201705-08910C

\section{Figures}




\section{Comparison of CMTs and mNGS}

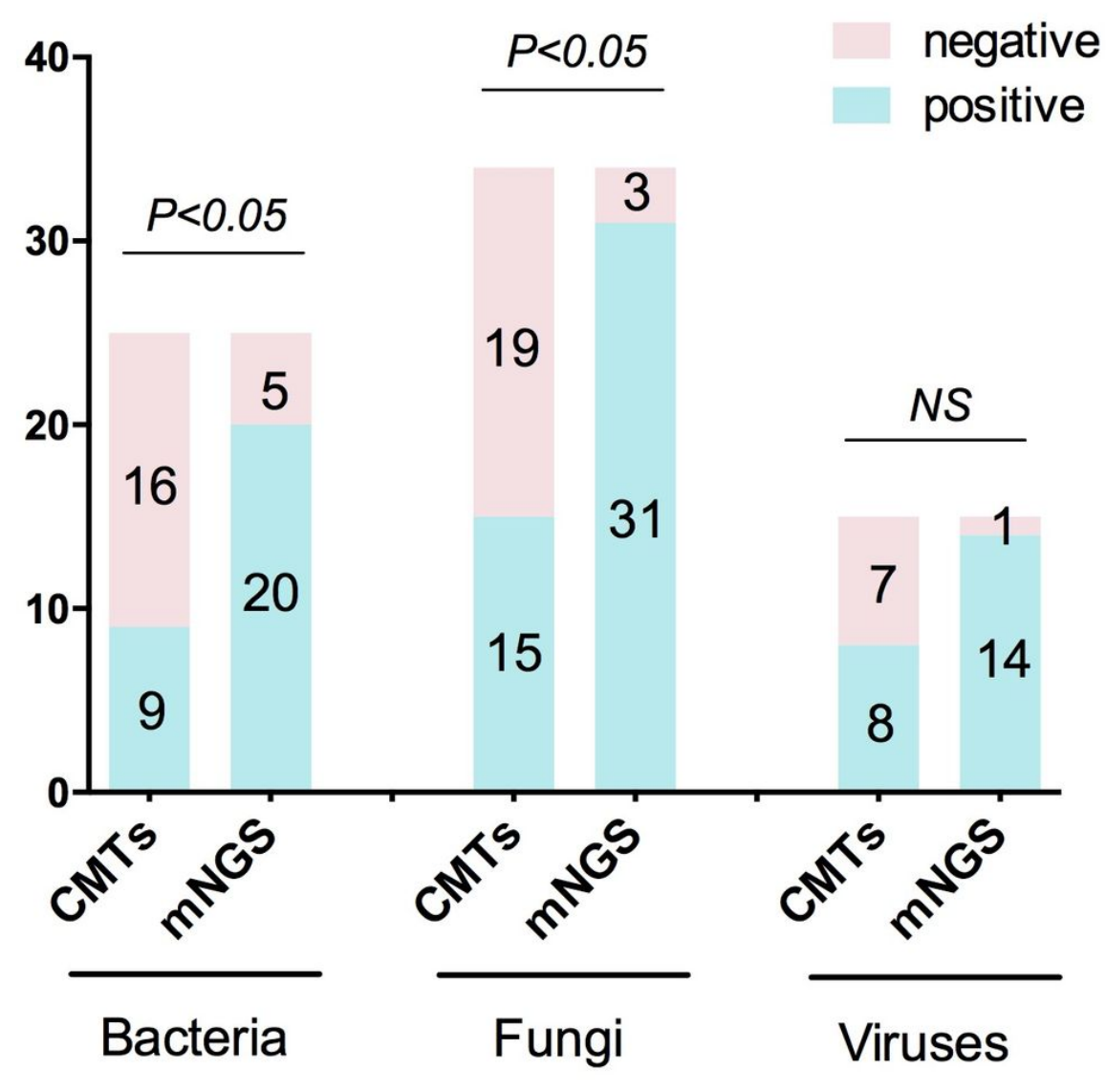

Figure 1

Comparison of CMTs and mNGS test for the different classes of pathogens.

Notes: The number of positive samples (y-axis) for pairwise mNGS and CMTs is plotted against the bacteria, fungus and virus groups (x-axis).

Abbreviations: mNGS: metagenomic next-generation sequencing;

CMTs: conventional microbiological tests 


\section{Distribution of Pathogens}

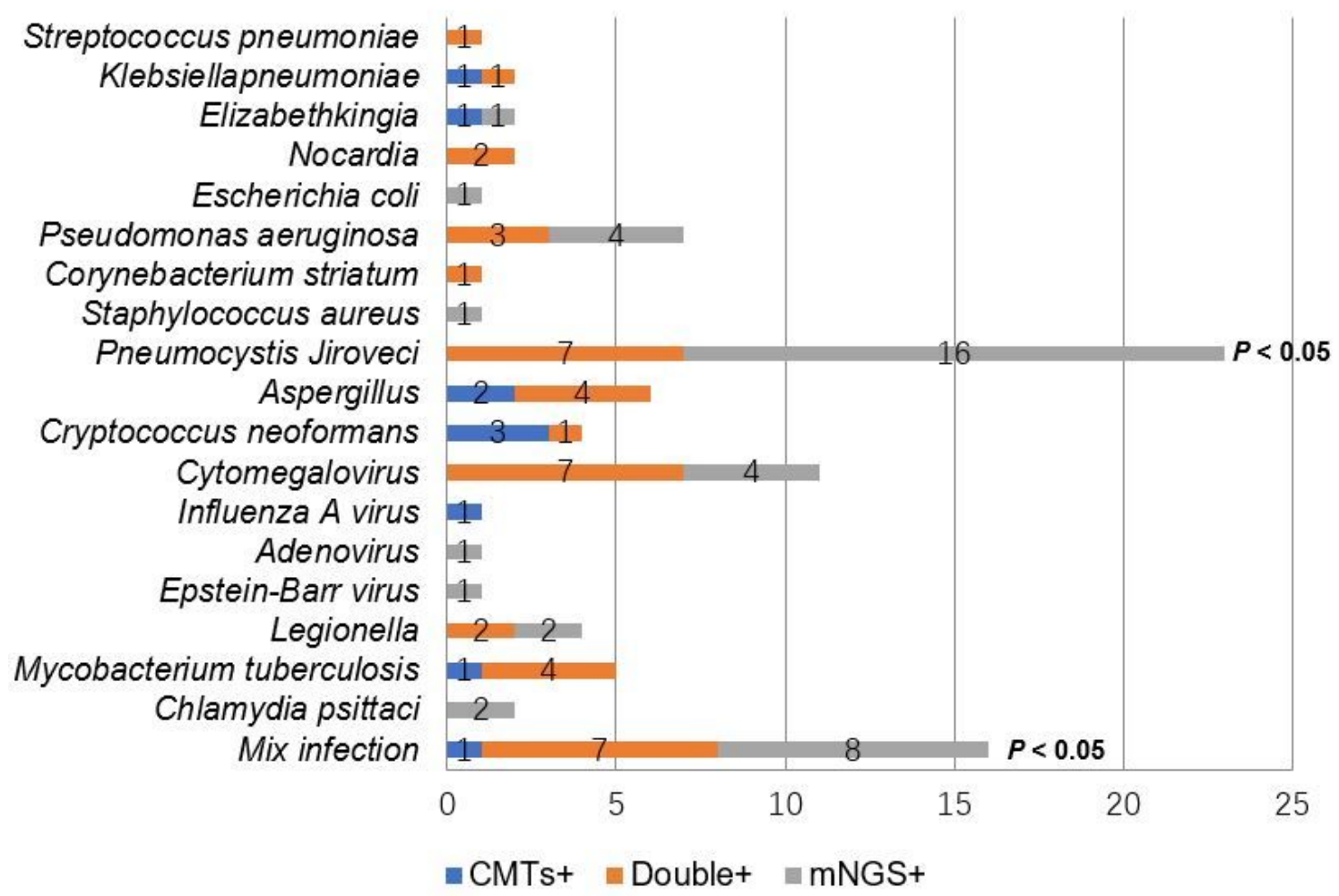

Figure 2

Distribution of pathogens identified in immunocompromised patients using CMTs versus mNGS.

Abbreviations: mNGS: metagenomic next-generation sequencing; CMTs: conventional microbiological tests; 


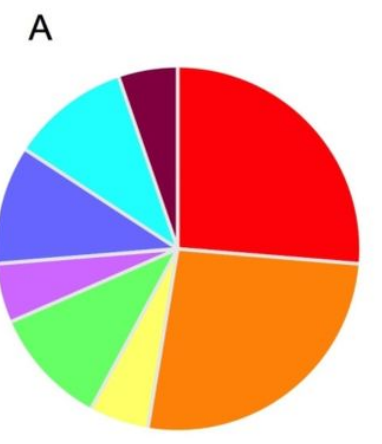

Bacteria, fungi and viruses, 5, 26.3\%

- Fungi and viruses, 5, 26.3\%

Fungi, viruses and legionella, 1, 5.3\%

Bacteria and fungi, 2, 10.5\%

Anaerobic bacteria, 1, 5.3\%

Fungi, 2, 10.5\%

Bacteria and TB, 2, 10.5\%

- Bacteria and viruses, $1,5.3 \%$

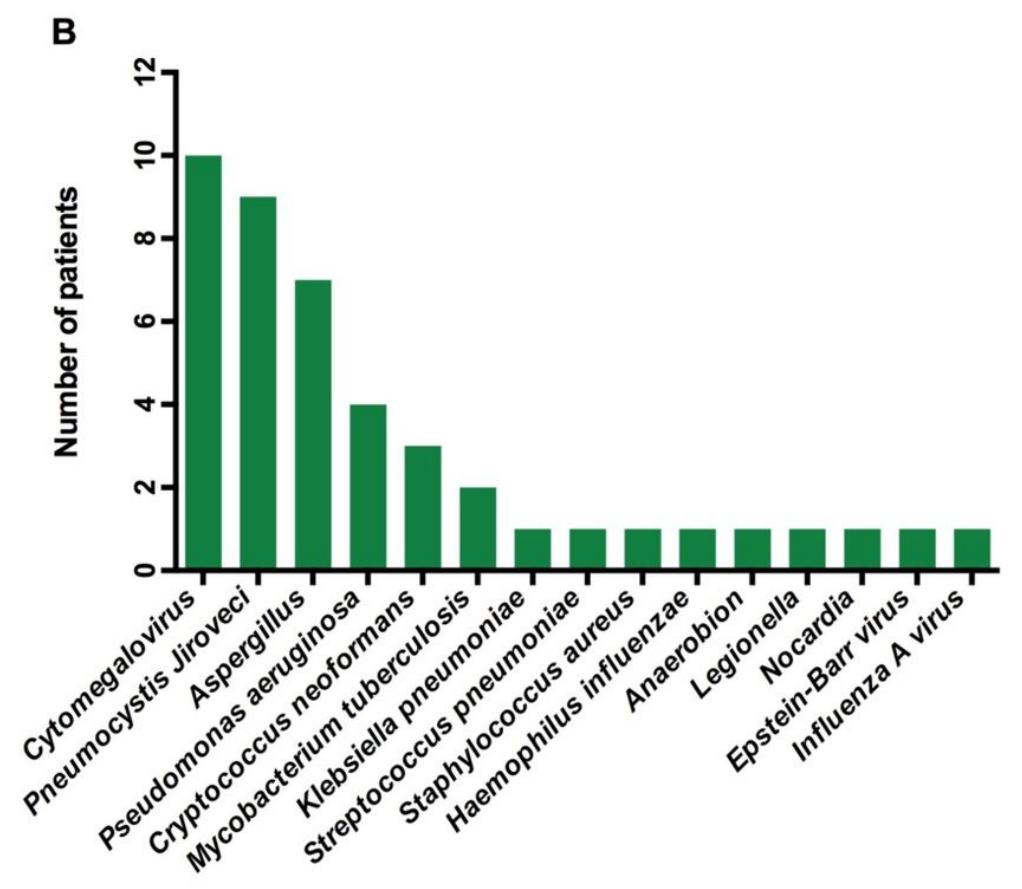

Figure 3

Distribution of pathogens identified in immunocompromised patients with mixed infection.

Notes: (A) Percentage of patients with mixed infection for various pathogens.

(B) Pathogen spectrum of immunocompromised patients with mixed infection.

Abbreviations: TB: Mycobacterium tuberculosis.

\section{Supplementary Files}

This is a list of supplementary files associated with this preprint. Click to download.

- Additionalfile1.xlsx 